\title{
Attacking the Network Time Protocol
}

\author{
Aanchal Malhotra, Isaac E. Cohen, Erik Brakke, and Sharon Goldberg \\ Boston University, Department of Computer Science \\ \{aanchal4, icohen93, ebrakke\}@bu.edu, goldbe@cs.bu.edu
}

\begin{abstract}
We explore the risk that network attackers can exploit unauthenticated Network Time Protocol (NTP) traffic to alter the time on client systems. We first discuss how an onpath attacker, that hijacks traffic to an NTP server, can quickly shift time on the server's clients. Then, we present an extremely low-rate (single packet) denial-of-service attack that an off-path attacker, located anywhere on the network, can use to disable NTP clock synchronization on a client. Next, we show how an off-path attacker can exploit IPv4 packet fragmentation to dramatically shift time on a client. We discuss the implications of these attacks on other core Internet protocols, quantify their attack surface using Internet measurements, and suggest a few simple countermeasures that can improve the security of NTP.
\end{abstract}

\section{INTRODUCTION}

NTP [42] is one of the Internet's oldest protocols, designed to synchronize time between computer systems communicating over unreliable variable-latency network paths. NTP has recently received some attention from security researchers due to software-implementation flaws [49], [57], and its potential to act as an amplifier for distributed denial of service (DDoS) attacks [14], [66]. However, the community still lacks visibility into the robustness of the NTP ecosystem itself, as well as the integrity of the timing information transmitted by NTP. These issues are particularly important because time is a fundamental building block for computing applications, and is heavily utilized by many cryptographic protocols.

NTP most commonly operates in an hierarchical clientserver fashion. Clients send queries to solicit timing information from a set of preconfigured servers that usually remain static over time. While NTP supports both symmetric and asymmetric cryptographic authentication [23], in practice, these modes of operation are rarely used (Section III).

Our goal is therefore to explore attacks on unauthenticated NTP that are possible within the NTP protocol specification [42]. We consider both (1) on-path attacks, where the attacker occupies a privileged position on the path between NTP client and one of its servers, or hijacks (with e.g., DNS [26], [27] or BGP [15], [21], [51]) traffic to the server, and (2) off-path attacks, where the attacker can be anywhere on the network and does not observe the traffic between client and any of its servers. This paper considers the following.

Permission to freely reproduce all or part of this paper for noncommercial purposes is granted provided that copies bear this notice and the full citation on the first page. Reproduction for commercial purposes is strictly prohibited without the prior written consent of the Internet Society, the first-named author (for reproduction of an entire paper only), and the author's employer if the paper was prepared within the scope of employment.

NDSS '16, 21-24 February 2016, San Diego, CA, USA

Copyright 2016 Internet Society, ISBN 1-891562-41-X

http://dx.doi.org/10.14722/ndss.2016.23090
Implications (Section II). We consider a few implications of attacks on NTP, highlighting protocols and applications whose correctness and security relies on accurate time.

On-path time-shifting attacks (Sections IV). We discuss how an on-path attacker can shift time on victim clients by hours or even years. Our attacks exploit NTP's behavior upon initialization, and the fact than an on-path attacker can trivially determine when an ntpd client is initializing. We also present a "small-step-big-step" attack (CVE-2015-5300) that stealthily shifts clocks when clients are unlikely to notice.

Off-path DoS attacks (Section V-C). We show how an off-path attacker can disable NTP at a victim client by exploiting NTP's rate-limiting mechanism, the Kiss-o'-Death $(K o D)$ packet.

1. DoS by Spoofed Kiss-o'-Death (CVE-2015-7704). We show how a single attacking machine can disable NTP on most of clients in the Internet. We find that ntpd versions earlier than 4.2.8p4 allow an off-path attacker to trivially spoof a KoD packet for each of the client's preconfigured servers; upon receipt of the spoofed KoD, the client stops querying its servers and stops updating its clock. Because the attacker only sends a few KoD packets per client, standard network scanning tools (nmap, zmap [18]) can be used to quickly launch this attack, in bulk, on all ntpd clients in the Internet. This vulnerability was patched in ntpd v4.2.8p4 following our work.

2. DoS by Priming the Pump (CVE-2015-7705). Even if KoD packets can no longer be trivially spoofed, an off-path attacker can still disable NTP at a victim client; this attack, however, requires the attacker to expend more resources (i.e., send more packets). Our off-path attacker sends the servers a high volume of queries that are spoofed to look like they come from the client. The servers then respond to any subsequent queries from the client with a valid KoD, and once again, the client stops querying its servers, and stops updating its local clock. Our recommended mitigations are in Section V-D

Off-path time-shifting attacks. Next, we consider off-path attackers that step time on victim NTP clients. We show how NTP's interaction with lower layer protocols (ICMP, IPv4) can be exploited in a new off-path IPv4 fragmentation attack that shifts time on a victim client. We explain why NTP's clock discipline algorithms require our attack to craft a stream of self-consistent packets (rather than just one packet, as in [26], [27]), and demonstrate its feasibility with a proofof-concept implementation. This attack, which has a small but non-negligible attack surface, exploits certain IPv4 fragmentation policies used by the server and client operating systems (Section VI-E), rather than specific issues with NTP.

Network measurements (Sections $I I I-B|V I-G| V I-H)$. The last measurement studies of the NTP ecosystem were conducted in 1999 [44] and 2006 [47], while a more recent study [14] 
focused on NTP DoS amplification attacks. We study the integrity of the NTP ecosystem using data from the openNTPproject [38], and new network-wide scans (Section [III-B].

Recommendations and disclosure. We began disclosing these results on August 20, 2015. The Network Time Foundation, NTPsec, Redhat's security team, and Cisco quickly patched their NTP implementations to prevent trivial spoofing of the KoD packet (CVE-2015-7704). We also worked with the openNTPproject to provide a resource that operators can use to measure their servers' vulnerability to our fragmentation attacks ${ }^{1}$ Our recommendations for hardening NTP are in Sections IV-C|V-D VI-I and summarized in Section VIII

Full version. The full version [36] of this paper has additional analysis and measurements that were omitted for brevity.

\section{WHY TIME MATTERS: \\ IMPLICATIONS OF ATTACKS ON NTP}

NTP lurks in the background of many systems; when NTP fails on the system, multiple applications on the system can fail, all at the same time. Such failures have happened. On November 19, 2012 [8], for example, two important NTP (stratum 1) servers, tick.usno.navy.mil and tock.usno.navy.mil went back in time by about 12 years, causing outages at a variety of devices including Active Directory (AD) authentication servers, PBXs and routers [46]. Exploits of individual NTP clients also serve as a building block for other attacks, as summarized in Table I] Consider the following:

TLS Certificates. Several authors [43, pg 33, pg 183] [29], [61] have observed that NTP could be used to undermine the security of TLS certificates, which are used to establish secure encrypted and authenticated connections. An NTP attacker that sends a client back in time could cause the host to accept certificates that the attacker fraudulently issued (that allow the attacker to decrypt the connection), and have since been revoked (For example, the client can be rolled back to mid-2014, when $>100 K$ certificates were revoked due to heartbleed [70].) Alternatively, an attacker can send the client back to a time when a certificate for a cryptographically-weak key was still valid. (For example, to 2008, when a bug in Debian OpenSSL caused thousands of certificates to be issued for keys with only 15-17 bits of entropy [19].) Moreover, most browsers today accept (non-root) certificates for 1024bit RSA keys, even though sources speculate that they can be cracked by well-funded adversaries [7]; thus, even a domain that revokes its old 1024-bit RSA certificates (or lets them expire) is vulnerable to cryptanalytic attacks when its clients are rolled back to a time when these certificates were valid. Some of these attacks were demonstrated by Selvi [61].

DNSSEC. DNSSEC provides cryptographic authentication of the Domain Name System (DNS) data. NTP can be used

\footnotetext{
${ }^{1}$ http://www.cs.bu.edu/ goldbe/NTPattack.html

${ }^{2}$ The attacker must also circumvent certificate revocation mechanisms, but several authors [28], [34], [48] point out that this is relatively easy to do in various settings. For instance, several major browsers rely on OCSP [58] to check if a certificate was revoked, and default to "soft-fail", i.e., accepting the certificate as valid, when they cannot connect to the OCSP server. NTP-based cache-flushing could also be useful for this purpose, by causing the client to 'forget' any old certificate revocation lists (CRLs) that it may have seen in the past; see also our discussion of routing attacks.
}

TABLE I. ATTACKING VARIOUS APPLICATIONS WITH NTP.

\begin{tabular}{|c|c|c|c|}
\hline To attack... & change time by ... & To attack... & change time by ... \\
\hline TLS Certs & years & Routing (RPKI) & days \\
\hline HSTS (see 60 & a year & Bitcoin (see [13]) & hours \\
\hline DNSSEC & months & API authentication & minutes \\
\hline DNS Caches & days & Kerberos & minutes \\
\hline
\end{tabular}

to attack a DNS resolver that performs 'strict' DNSSEC validation, i.e., fails to return responses to queries that fail cryptographic DNSSEC validation. An NTP attack that sends a resolver forward in time will cause all timestamps on DNSSEC cryptographic keys and signatures to expire (the recommended lifetime for zone-signing keys in DNSSEC is 1 month [33]); the resolver and all its clients thus lose connectivity to any domain secured with DNSSEC. Alternatively, an NTP attack that sends a resolver back in time allows for DNSSEC replay attacks; the attacker, for example, roll to a time in which a certain DNSSEC record for a domain name did not exist, causing the resolver to lose connectivity to that domain. Since the recommended lifetime for DNSSEC signatures is no more than 30 days [33], this attack would need to send the resolver back in time by a month (or more, if the time in which the DNSSEC record did not exist was further in the past).

Cache-flushing attacks. NTP can be used to flush caches. The DNS, or example, uses caching to minimize the number of DNS queries a resolver makes to a public nameserver, thus limiting network traffic. DNS cache entries typically live for around 24 hours, so rolling a resolver forward in time by a day would cause most of its cache entries to expire [29], [43]. A widespread NTP failure (like the one in November 2012) could cause multiple resolvers to flush their caches all at once, simultaneously flooding the network with DNS queries.

Interdomain routing. NTP can be used to exploit the Resource Public Key Infrastructure (RPKI) [35], a new infrastructure for securing routing with BGP. The RPKI uses Route Origin Authorizations (ROAs) to cryptographically authenticate the allocation of IP address blocks to networks. ROAs prevent hijackers from announcing routes to IP addresses that are not allocated to their networks. If a valid ROA is missing, a 'relying party' (that relies on the RPKI to make routing decisions) can lose connectivity to the IPs in the missing ROA $3^{3}$ As such, relying parties must always download a complete set of valid ROAs; to do this, they verify that they have downloaded all the files listed in cryptographically-signed 'manifest' files. To prevent the relying party from rolling back to a stale manifest that might be missing a ROA, manifests have monotonicallyincreasing 'manifest-numbers', and typically expire within a day [25]. NTP attacks, however, can first roll the relying party forward in time, flushing its cache and causing it to 'forget' its current manifest-number, and then roll the relying party back in time, so that it accepts a stale manifest as valid.

Authentication. Various services [4], [16] expose APIs that require authentication each time an application queries them. To prevent replay attacks, queries require a timestamp that is within some short window of the server's local time, see e.g., [24. Sec 3.3]; Amazon S3, for example, uses a 15-minute window. An NTP attacker can also launch replay attacks on Kerberos, which requires clients to present tickets which have been timestamped within minutes [32].

\footnotetext{
${ }^{3}$ See [12. Side Effect 6]: the relying party loses connectivity if it uses 'drop invalid' routing policy [12 Sec. 5], and the missing ROA has 'covering ROA'.
} 


\section{THE NTP ECOSYSTEM}

We start with background on the NTP protocol, and use a measurement study to discuss its structure and topology. NTP has evolved in more fluid fashion than other core Internet protocols like DNS or BGP. While NTP is described in RFC 5905 [42], practically speaking, the protocol is determined by the NTP reference implementation $n t p d$, which has changed frequently over the last decades [66]. (For example, root distance $\Lambda$ (equation (4)) is a fundamental NTP parameter, but is defined differently in RFC 5905 [42, Appendix A.5.5.2], ntpd v4.2.6 (the second most popular version of ntpd we found in the wild) and ntpd v4.2.8 (the latest version).)

\section{A. Background: The NTP Protocol.}

NTP most commonly operates in an hierarchical clientserver fashion ${ }^{4}$ Clients send queries to solicit timing information from a set of servers. This set of servers is manually configured before the client initializes and remains static over time. In general, the ntpd client can be configured with up to 10 servers. Online resources suggest configuring anywhere from three to five servers [31], and certain OSes (e.g., MAC OS $\mathrm{X}$ 10.9.5) default to installing ntpd with exactly one server (i.e., time.apple.com). At the root of the NTP hierarchy are stratum 1 NTP servers, that provide timing information to stratum 2 client systems. Stratum 2 systems provide time to stratum 3 systems, and so on, until stratum 15 . Stratums 0 and 16 indicate that a system is unsynchronized. NTP servers with low stratum often provide time to the Internet at large (e.g., pool.ntp.org. tick.usno.navy.mil); our organization, for example, has stratum 2 servers that provide time to internal stratum 3 machines, and take time from public stratum 1 servers.

Client/server communications. An NTP client and server periodically exchange a pair of messages; the client sends the server a mode 3 NTP query and the server responds with a mode 4 NTP response. This two-message exchange uses the IPv4 packet shown in Figure 1 and induces the following four important timestamps on the mode 4 response:

$\begin{array}{ll}T_{1} & \begin{array}{l}\text { Origin timestamp. Client's system time when client } \\ \text { sent mode } 3 \text { query. }\end{array} \\ T_{2} & \begin{array}{l}\text { Receive timestamp. Server's system time when server } \\ \text { received mode } 3 \text { query. }\end{array} \\ T_{3} & \begin{array}{l}\text { Transmit timestamp. Server's system time when } \\ \text { server sent mode } 4 \text { response. }\end{array} \\ T_{4} & \begin{array}{l}\text { Destination timestamp. Client's system time when } \\ \text { client received mode } 4 \text { response. (Not in packet.) }\end{array}\end{array}$

The round-trip delay $\delta$ during the exchange is therefore:

$$
\delta=\left(T_{4}-T_{1}\right)-\left(T_{3}-T_{2}\right)
$$

Offset $\theta$ quantifies the time shift between a client's clock and a server's clock. Assume that delays on the forward (client $\rightarrow$ server) and reverse (server $\rightarrow$ client) network paths are symmetric and equal to $\frac{\delta}{2}$. Then, the gap between the server and client clock is $T_{2}-\left(T_{1}+\frac{\delta}{2}\right)$ for the mode 3 query, and

\footnotetext{
${ }^{4}$ NTP also supports several less popular modes including broadcast, where a set of clients listen to a server that broadcasts timing information, and symmetric peering, where servers (typically at the same stratum) exchange time information. We only consider client-server mode.
}

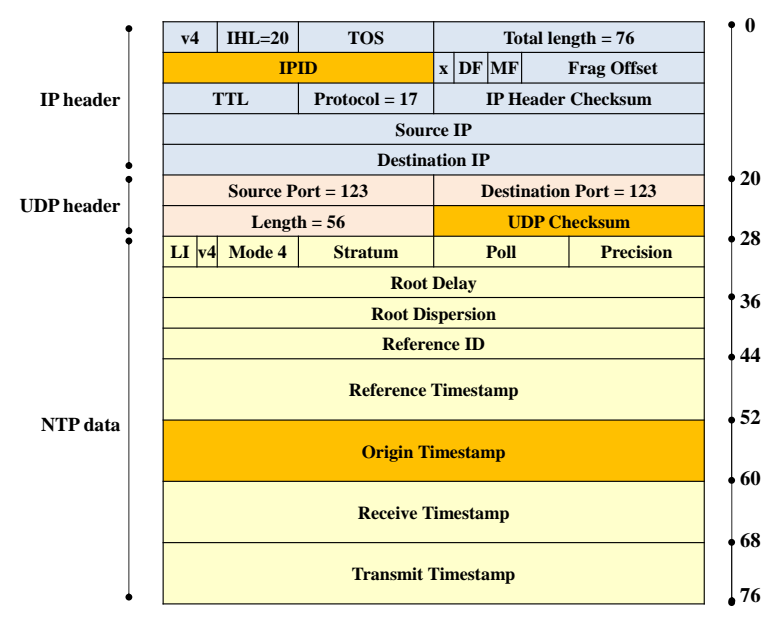

Fig. 1. Mode 4 NTP Packet, highlighting nonces and checksums.

$T_{3}-\left(T_{4}-\frac{\delta}{2}\right)$ for the mode 4 response. Averaging these two quantities gives the offset:

$$
\theta=\frac{1}{2}\left(\left(T_{2}-T_{1}\right)+\left(T_{3}-T_{4}\right)\right)
$$

An NTP client adaptively and infrequently selects a single server (from its set of pre-configured servers) from which it will take time. The IPv4 address of the selected server is recorded in the reference ID field of every NTP packet a system sends, and the reference timestamp field records the last time it synchronized to its reference ID. Notice that this means that any client querying a server $S_{2}$ can identify exactly which IPv4 NTP server $S_{1}$ the server $S_{2}$ has used for synchronization.

Infrequent clock updates. NTP infrequently updates a client's clock because (1) a client and server must exchange between eight to hundreds of messages before the client's clock discipline algorithms synchronizes it to the server [42, Sec. 10-12] and (2) messages are exchanged at infrequent polling intervals (on the order of minutes) that are adaptively chosen by a randomized poll process [42. Sec. 13].

Authentication. How does the client know that it's talking to its real NTP server and not to an attacker? While NTPv4 supports both symmetric and asymmetric cryptographic authentication, this is rarely used in practice. Symmetric cryptographic authentication appends an MD5 hash keyed with symmetric key $k$ of the NTP packet contents $m$ as MD5 $(k \| m)$ [43, pg 264] to the NTP packet in Figure 11. The symmetric key must be pre-configured manually, which makes this solution quite cumbersome for public servers that must accept queries from arbitrary clients. (NIST operates important public stratum 1 servers and distributes symmetric keys only to users that register, once per year, via US mail or facsimile [3]; the US Naval Office does something similar [2].) Asymmetric cryptographic authentication is provided by the Autokey protocol, described in RFC 5906 [23]. RFC 5906 is not a standards-track document (it is classified as 'Informational'), NTP clients do not request Autokey associations by default [1], and many public NTP servers do not support Autokey (e.g., the NIST timeservers [3], many servers in pool.ntp.org). In fact, a lead developer of the ntpd client wrote in 2015 [64]: "Nobody should be using autokey. Or from the other direction, if you are using autokey you should stop using it." For the remainder of this paper, we shall assume that NTP messages are unauthenticated. 
TABLE II. TOP NTPD VERSIONS IN $r v$ DATA FROM MAY 2015.

\begin{tabular}{|c|c|c|c|c|c|c|c|c|c|}
\hline ntpd version & 4.1 .1 & 4.2 .6 & 4.1 .0 & 4.2 .4 & 4.2 .0 & 4.2 .7 & 4.2 .8 & 4.2 .5 & 4.4 .2 \\
\hline \# servers & $1,984,571$ & 702,049 & 216,431 & 132,164 & 100,689 & 38,879 & 35,647 & 20,745 & 15,901 \\
\hline
\end{tabular}

TABLE III. TOP OSES IN $r v$ DATA FROM MAY 2015

\begin{tabular}{|c|c|c|c|c|c|c|c|c|c|}
\hline OS & Unix & Cisco & Linux & BSD & Junos & Sun & Darwin & Vmkernal & Windows \\
\hline \# servers & $1,820,957$ & $1,602,993$ & 835,779 & 38,188 & 12,779 & 6,021 & 3625 & 1994 & 1929 \\
\hline
\end{tabular}

\section{B. Measuring the NTP ecosystem.}

We briefly discuss the status of today's NTP ecosystem. Our measurement study starts by discovering IP addresses of NTP servers in the wild. We ran a zmap [18] scan of the IPv4 address space using mode 3 NTP queries on April 12-22, 2015, obtaining mode 4 responses from 10,110,131 IPs.We augmented our data with openNTPproject [38] data from JanuaryMay 2015, which runs weekly scans to determine which IPs respond to NTP control queries. (These scans are designed to identify potential DDoS amplifiers that send large packets in response to short control queries [14].) The openNTPproject logs responses to NTP read variable ( $r v$ ) control queries. $r v$ responses provide a trove of useful information including: the server's OS (also useful for OS fingerprinting!), its ntpd version, its reference ID, and more. Merging our zmap data with the openNTPproject $r v$ data gave a total of 11,728,656 IPs that potentially run NTP servers.

OSes and clients in the wild. We use openNTPproject's $r v$ data to get a sense of the OSes and ntpd clients that are present in the wild. Importantly, the $r v$ data is incomplete; $r v$ queries may be dropped by firewalls and other middleboxes, NTP clients can be configured to refuse these queries, and some $r v$ responses omit information. (This is why we had only 4M IPs in the $r v$ data, while 10M IPs responded to our mode 3 zmap scan.) Nevertheless, we get some sense of what systems are out there by looking at the set of $r v$ responses from May 2015. In terms of operating systems, Table III shows many servers running Unix, Cisco or Linux. Table $\overline{\mathrm{IV}}$ indicates that Linux kernels are commonly v2 (rather the more recent v3); in fact, Linux v3.0.8 was only the $13^{\text {th }}$ most popular Linux kernel. Meanwhile, Table II shows that ntpd v4.1.1 (released 2001) and v4.2.6 (released 2008) are most popular; the current release v4.2.8 (released 2014) is ranked only $8^{\text {th }}$ amongst the systems we see. The bottom line is that there are plenty of legacy NTP systems in the wild. As such, our lab experiments and attacks study the behavior of two NTP reference implementations: ntpd v4.2.6p5 (the second most popular version in our dataset) and ntpd v4.2.8p2 (the latest release as of May 2015).

Bad timekeepers. Next, we used our mode 3 zmap data to determine how many bad timekeepers-servers that are unfit to provide time - are seen in the wild. To do this, we compute the offset $\theta$ (equation (2)) for each IP that responded to our mode 3 queries, taking $T_{1}$ from the Ethernet frame time of the mode 3 query, $T_{4}$ from the Ethernet frame time of the mode 4 query, and $T_{2}$ and $T_{3}$ from the mode 4 NTP payload. We found many bad timekeepers $-1.7 \mathrm{M}$ had $\theta \geq 10 \mathrm{sec}, 3.2 \mathrm{M}$ had stratum 0 or 16 , and the union of both gives us a total of $3.7 \mathrm{M}$ bad timekeepers. Under normal conditions, NTP is great at discarding information from bad timekeepers, so it's unlikely that most of these servers are harming anyone other than themselves; we look into this in the full version [36].
TABLE IV. TOP LINUX KERNELS IN $r v$ DATA FROM MAY 2015.

\begin{tabular}{|c|c|c|c|c|c|c|c|c|c|}
\hline kernel & 2.6 .18 & 2.4 .23 & 2.6 .32 & 2.4 .20 & 2.6 .19 & 2.4 .18 & 2.6 .27 & 2.6 .36 & 2.2 .13 \\
\hline \# servers & 123,780 & 108,828 & 97,168 & 90,025 & 71,581 & 68,583 & 61,301 & 45,055 & 29550 \\
\hline
\end{tabular}

TABLE V. STRATUM DistRIBUTION IN OUR DATASET.

\begin{tabular}{|c|c|c|c|c|c|c|c|c|c|}
\hline stratum & 0,16 & 1 & 2 & 3 & 4 & 5 & 6 & $7-10$ & $11-15$ \\
\hline
\end{tabular}

\begin{tabular}{|l|l|l|l|l|l|l|l|l|}
\hline \# servers $3,176,142$ & 115,357 & $1,947,776$ & $5,354,922$ & $1,277,942$ & 615,633 & 162,162 & 218,370 & 187,348 \\
\hline
\end{tabular}

Topology. Since a system's reference ID reveals the server from which it takes time, our scans allowed us to start building a subset of the NTP's hierarchical client-server topology. However, a reference ID only provide information about one of a client's preconfigured servers. In an effort to learn more, on June 28-30, 2015 we used nmap to send an additional mode 3 NTP query to every IP that had only one parent server in our topology; merging this with our existing data gave us a total of $13,076,290$ IPs that potentially run NTP servers. We also wanted to learn more about the clients that synchronize to bad timekeepers. Thus, on July 1, 2015, we used the openNTPproject's scanning infrastructure to send a monlist query to each of the $1.7 \mathrm{M}$ servers with $\theta>10$ sec. While monlist responses are now deactivated by many servers, because they have been used in DDoS amplification attacks [14], we did obtain responses from 22,230 of these bad timekeepers. Monlist responses are a trove of information, listing all IPs that had recently sent NTP packets (of any mode) to the server. Extracting only the mode 3 and 4 data from each monlist response, and combining it with our existing data, gave us a total of 13,099,361 potential NTP servers.

Stratum. Table $\mathrm{V}$ shows the distribution of stratums in our entire dataset. Note that there is not a one-to-one mapping between an NTP client and its stratum; because an NTP client can be configured with servers of various stratum, the client's stratum can change depending on the server it selects for synchronization. Thus, TableV Vpresents the 'best' (i.e., lowest) stratum for each IP in our dataset. Unsurprisingly, stratum 3 is most common, and, like [14] we find many unsynchronized (stratum 0 or 16) servers.

Degree distribution. Figure 2 shows the client (i.e., child) degree distribution of the servers in our topology. We note that our topology is highly incomplete; it excludes information about clients behind a NAT or firewall, as well as servers that a client is configured for but not synchronized to ${ }^{5}$ The degree distribution is highly skewed. Of $13.1 \mathrm{M}$ IPs in our dataset, about $3.7 \mathrm{M}(27.8 \%)$ had clients below them in the

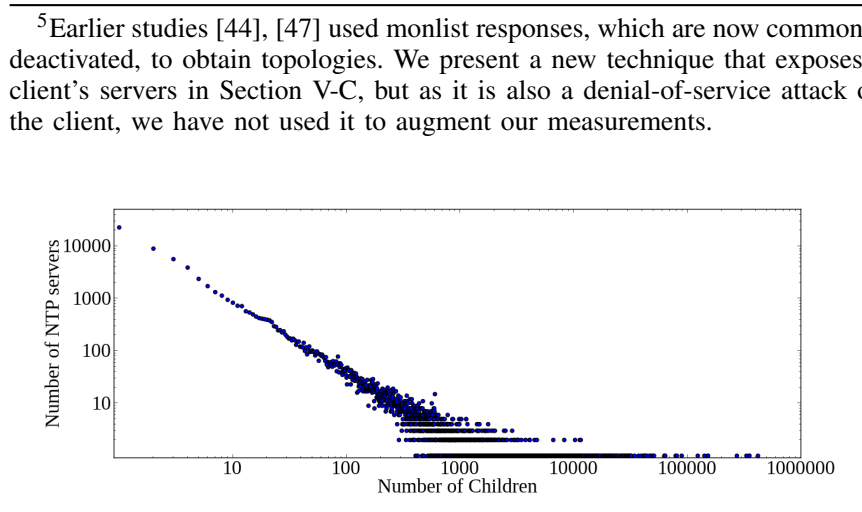

Fig. 2. Client-degree distribution of NTP servers in our dataset; we omit servers with no clients. 
NTP hierarchy. Of these 3.7M servers with clients, $99.4 \%$ of them have fewer than 10 clients, while only $0.2 \%$ of them have more than 100 clients. However, servers with more than 100 client tend to have many clients, averaging above $1.5 \mathrm{~K}$ clients per server, with the top 50 servers having at least $24.5 \mathrm{~K}$ clients each. Compromising these important servers (or hijacking their traffic) can impact large swaths of the NTP ecosystem.

\section{HOW TO STEP TIME WITH NTP.}

Unauthenticated NTP traffic is vulnerable to on-path attacks, as was pointed out by various authors [23], [29], [45], [60], [61]. While on-path attacks are sometimes dismissed because the attacker requires a privileged position on the network, it is important to remember that an attacker can use various hijacking techniques to place herself on the path to an NTP server. For instance, ntpd configuration files allow clients to name servers by either their IP or their hostname (e.g., MAC OS X 10.9.5 comes with an NTP client that is preconfigured to take time from the host time.apple.com. while many systems rely on the pool of servers that share the hostname pool.ntp.org). By hijacking the DNS entries for these hostnames [26], [27], an attacker can quietly manipulate the NTP traffic they send. Moreover, NTP relies on the correctness of IP addresses; thus ARP-spoofing in a local-area network or even global attacks on BGP [21] (like those seen in the wild [15], [51]) can divert NTP traffic to an attacker.

In Section $\Pi$ and Table $\Pi$ we saw that dramatic shifts in time (years, months) are required when NTP attacks are used inside larger, more nefarious attacks. Can an on-path attacker really cause NTP clients to accept such dramatic shifts in time?

\section{A. Time skimming}

At first glance, the answer should be no. NTP defines a value called the panic threshold which is $1000 \mathrm{sec}$ (about 16 minutes) by default; if NTP attempts to tell the client to alter its local clock by a value that exceeds the panic threshold, then the NTP client "SHOULD exit with a diagnostic message to the system log" [42]. Our experiments confirm that ntpd v4.2.6 and v4.2.8 quit when they are initially synchronized to a server that then starts to offer time that exceeds the panic threshold.

One way to circumvent this is through an adaption of [60]'s "time-skimming" technique ${ }^{6}$ so that the man-in-the-middle slowly steps the client's local clock back/forward in steps smaller than the panic threshold. However, this comes with a caveat: it can take minutes or hours for ntpd to update a client's local clock. To understand why, observe that in addition to the panic threshold, NTP also defines a step threshold of $125 \mathrm{~ms}$ [42]. A client will accept a time step larger than step threshold but smaller than the panic threshold as long as at least "stepout" seconds have elapsed since its last clock update; the stepout value is 900 seconds (15 minutes) in ntpd v4.2.6 and RFC 5905 [42], and was reduced to 300 seconds (5 minutes) in ntpd v4.2.8. Thus, shifting by one year using steps of size 16 minute each requires $\frac{1 \times 365 \times 24 \times 60}{16}=33 \mathrm{~K}$ total steps; with a 5-minute stepout, this takes at least 114 days.

\footnotetext{
${ }^{6}$ Selvi's 60 time-skimming attack allows for fast timesteps on clients that update their clocks at predictable intervals-for instance, Fedora Linux sends a query every minute and updates its clock immediately upon receipt of the response. The full ntpd implementation has more complex clock update mechanisms that thwart this attack. (See e.g., TEST11 in Section VI-D)
}

\section{B. Exploiting reboot.}

There are other ways to quickly shift a client's time. ntpd has a configuration option called $-g$, which allows an NTP client that first initializes (i.e., before it has synchronized to any time source) to accept any time shift, even one exceeding the panic threshold. (We have confirmed that both ntpd v4.2.6p5 and ntpd v4.2.8p2 on Ubuntu13.16.0-24-generic accept a single step 10 years back in time, and forward in time, upon reboot.) The $-g$ configuration is quite natural for clocks that drift significantly when systems are powered down, and many OSes, including Linux, do run ntpd with $-g$ by default.

Reboot. An on-path attacker can exploit - $g$ by waiting until the client restarts as a result of power cycling, software updates, or other 'natural events'. Importantly, the attacker knows exactly when the client restarts, because the client puts 'INIT' in the reference ID of its packets (Figure 1), including the mode 3 queries the client sends the server. Moreover, a determined attacker can deliberately cause ntpd to restart using e.g., a packet-of-death like Teardrop [9].

Feel free to panic. Suppose, on the other hand, that an NTP attacker shifts a client's time beyond the panic threshold, causing the client to quit. If the operating system is configured to reboot the NTP client, the rebooted NTP client will initialize and accept whatever (bogus) time it obtains from its NTP servers. Indeed, this seems to have happened with some OSes during the November 2012 NTP incident [39].

Small-step-big-step. Users might notice strange shifts in time if they occur immediately upon reboot. However, we have found that ntpd allows an on-path attacker to shift time when clients are less likely to notice. To understand how, we need to look into ntpd's implementation of the $-g$ configuration.

Small-step-big-step with ntpd v4.2.6. One might expect -g to allow for timesteps that exceed the panic threshold only upon initialization-when the client updates its clock for the very first time upon starting. To implement this, ntpd allows steps exceeding the panic threshold only when a variable called allow_panic is TRUE. ntpd v4.2.6p5 sets allow_panic to TRUE only upon initialization with the $-g$ configuration (otherwise, it is initialized to FALSE), and set to FALSE if the client (1) is just about to update its local clock by a value less than the step threshold $(125 \mathrm{~ms})$, and (2) is already in a state called SYNC, which means it recently updated its clock by a value less than the step threshold. Normally, a client initializes and (1) and (2) occur after two clock updates. However, if an attacker is able to prevent the client from making ever two contiguous clock updates (one after the other) of less than 125 ms each, then allow_panic remains TRUE.

The following small-step-big-step attack on ntpd v4.2.6 exploits the above observation. First, the client reboots and begins initializing; it signals this to the server by putting 'INIT' in the reference ID of its mode 3 queries [42, Fig. 13]). Next, the client synchronizes to the server; it signals this with the server's IP address in the reference ID of its mode 3 queries [42, Fig. 13]). When the server sees that the client has synchronized once, the server sends the client a 'small step' greater than the STEP threshold $(125 \mathrm{~ms})$ and less than the panic threshold $(\approx 16 \mathrm{~min})$; the client signals that it has accepted this timestep by putting 'STEP' in its reference ID [42, Fig. 13]). When the server sees that the client is in 
'STEP' mode, the server immediately sends the client a 'big step' that exceeds the panic threshold. At this point, the client does not panic, because it never set allow_panic to FALSE. Indeed, one might even interpret this as expected behavior per RFC 5905 [42]:

STEP means the offset is less than the panic threshold, but greater than the step threshold STEPT (125 $\mathrm{ms})$. In this case, the clock is stepped to the correct offset, but ... all associations MUST be reset and the client begins as at initial start.

Small-step-big-step with ntpd v4.2.8. Meanwhile, ntpd v4.2.8p2 sets allow_panic to FALSE under conditions (1) and (2), OR if (1) holds and (3) the client is in FSET state, which is the state the client enters upon initialization. Normally, a client initializes and (1) and (3) occur after one clock update. Thus, small-step-big-step succeeds iff every clock update the client receives exceeds the step threshold (125ms).

Stealthy time shift. As an application of the small-step-bigstep attack, an on-path attacker can preform a bogus big step and then to quickly bring the client's clock back to normal, so that the client never notices; this might be useful for stealthily flushing a client's cache, or expiring certain cryptographic objects (see Section III). To do this, the attacker waits for ntpd to reboot (or deliberately causes a reboot), and ensures that every clock update made by the client makes is larger than 125 $\mathrm{ms}$, sending the client into STEP mode. To keep things stealthy, the attacker can e.g., first shift the client forward by $150 \mathrm{~ms}$, then back by $150 \mathrm{~ms}$, then forward by $150 \mathrm{~ms}$, etc.. Then, when the attacker is ready, it can send a big step that exceeds the panic threshold, perform nefarious deeds, and finally send another big step that sets the client's clock back to normal.

\section{Recommendation: Careful with -9}

The security of ntpd should not rely on $100 \%$ OS uptime, so users should be careful with the $-g$ option. One solution is to not use the $-g$ option. Alternatively, one can detect feel-free-to-panic attacks by monitoring the system $\log$ for panic events and being careful when automatically restarting ntpd after it quits. Monitoring should also be used to detect suspicious reboots of the OS (that might indicate the presence of a small-step-big-step or other reboot-based on-path attacks). Implementors can prevent small-step-big-step attacks by patching ntpd to ensure that the allow_panic variable is set to FALSE after the very first clock update upon initialization; this issue has been captured in CVE-2015-5300.

\section{KISS-O'-DEATH: \\ OFF-PATH DENIAL-OF-SERVICE ATtACKS.}

We show how NTP security can be stymied by another aspect of the NTP protocol: the 'Kiss-o-death' (KoD) packet. KoD packets are designed to reduce load at an NTP server by rate-limiting clients that query the server too frequently; upon receipt of a KoD from its server, the client refrains from querying that server for some period of time [42, Sec 7.4]. We now show how KoD packets can be used to launch off-path denial-of-service attacks on NTP clients.

\section{A. Why are off-path attacks hard?}

We first need to understand why it is usually difficult to spoof NTP mode 4 packets (Figure 1) from off-path.

TEST2: The origin timestamp. Like many other protocols, NTP requires clients to check that a nonce in the client's query matches a nonce in the server's response; that way, an off-path attacker, that cannot observe client-server communications, does not know the nonce and thus has difficulty spoofing the packet. (This is analogous to source port randomization in TCP/UDP, sequence number randomization in TCP, and transaction ID randomization in DNS.) NTP uses the origin timestamp as a nonce: the client checks that (a) the origin timestamp on the mode 4 response sent from server to client (Figure 11), matches (b) the client's local time when he sent the corresponding mode 3 query, which is sent in the transmit timestamp field of the mode 3 query sent from client to server. This is called TEST 2 in the ntpd code. (Note that ntpd does not randomize the UDP source port to create an additional nonce; instead, all NTP packets have UDP source port 123.)

How much entropy is in NTP's nonce? The origin timestamp is a 64 bit value, where the first 32 bits represent seconds elapsed since January 1, 1900, and the last 32 bits represent fractional seconds. A client whose system clock has e.g., $\rho=-12$ bit precision $\left(2^{-12}=244 \mu \mathrm{s}\right)$ puts a $32-12=20$ bit random value in the least significant bit of the timestamp. Thus, for precision $\rho$, the origin timestamp has at least $32+\rho$ bits of entropy. However, because polling intervals are no shorter than 16 seconds [42], an off-path attacker is unlikely to know exactly when the client sent its mode 3 query. We therefore suppose that the origin timestamp has about 32 bits of entropy. This is a lot of entropy, so one might conclude that NTP is robust to off-path attacks. However, in this section and Section VI, we will show that this is not the case.

\section{B. Exploiting the Kiss-O'-Death Packet}

A server sends a client a Kiss-O'-Death (KoD) if a client queries it too many times within a specified time interval; the parameters for sending KoD are server dependent. The KoD is an NTP packet (Figure 1) with mode 4, leap indicator 3, stratum 0 and an ASCII 'kiss code' string in the reference ID field. According to RFC5905 [42]:

For kiss code RATE, the client MUST immediately reduce its polling interval to that server and continue to reduce it each time it receives a RATE kiss code.

In ntpd v4.2.6 and v4.2.8, this is implemented by having the client stop querying the server for a period that is at least as long as the poll value field in the received KoD packet ${ }^{7}$ Our experiments confirm that if the KoD packet has polling interval $\tau_{\text {kod }}=17$ then the ntpd $\mathrm{v} 4.2 .8$ client will stop querying the server for at least $2^{\tau_{\text {kod }}} \sec (36$ hours). The poll field in the NTP packet is an 8-bit value (i.e., ranging from 0 to 255), but RFC 5905 [42, pg 10] defines the maximum allowable

\footnotetext{
${ }^{7}$ Interestingly, RFC 5905 [42 Sec. 7.4] defines an even more dangerous type of KoD packet: " For kiss codes DENY and RSTR, the client MUST demobilize any associations to that server and stop sending packets to that server". Thus, spoofing a single DENY or RSTR KoD packet can completely disconnect a client from its server! Fortunately, however, neither ntpd v4.2.6 or v4.2.8 honor this functionality.
} 
poll value to be 17 . The most recent ntpd implementation, however, will accept KoDs with poll values even larger than 17 ; setting $\tau_{\text {kod }}=25$, for example, should cause the client to stop querying its server for at least $2^{25}$ seconds, i.e., $\approx 1$ year.

Spoofing a KoD from off-path. How does the client know that the KoD packet came from the legitimate server, and not from an attacker? With regular mode 4 responses, the client uses the origin timestamp as a nonce. While it seems reasonable to expect this check to be performed on the KoD packet as well, RFC 5905 [42, Sec. 7.4] does not seem to explicitly require this. Moreover, lab experiments with ntpd v4.2.6p5 and $\mathrm{v} 4.2 .8 \mathrm{p} 3$ show that the client accepts a KoD even if its origin timestamp is bogus. This means that an offpath attacker can trivially send the client a $\mathrm{KoD}$ that is spoofed to look like it came from its server; the only information the attacker needs is the IP addresses of the relevant client and server. Moreover, by setting the poll value in the spoofed $\mathrm{KoD}$ to be an unreasonably high value $\left(e . g ., \tau_{\text {kod }}=25\right)$, the spoofed KoD will prevent the client from querying its server for an extended period of time. This vulnerability, captured in CVE-2015-7704, was patched in ntpd v4.2.8p4 after the disclosure of our work.

Eliciting a KoD from off-path: Priming the pump. Even if the client does validate the origin timestamp on the KoD packet, an off-path attacker could still elicit a valid KoD packet for the client from the server. To do this, the off-path attacker 'primes-the-pump' at the server, by sending multiple mode 3 queries spoofed to look like they come from the victim client; the server then 'gets angry' at the client, and responds to the client's legitimate mode 3 queries with a KoD. The client will accept this $\mathrm{KoD}$ because it has a valid origin timestamp (matching that in the client's legitimate query). This issue has been captured in CVE-2015-7705. Interestingly, recent NTP security bulletins (as of 09/2015) have increased this attack surface by recommending that servers send KoDs [5].

Attack efficiency. A single spoofed KoD packet with high poll (e.g., $\left.\tau_{\text {kod }} \geq 25\right)$ can essentially prevent the client from ever taking time from its server, and thus gives rise to a very low-rate off-path denial-of-service attack (CVE-2015-7704). Meanwhile, eliciting a KoD by priming-the-pump (CVE-20157705) requires more packets, because:

1) The attacker must send several packets to the server to elicit the KoD, and

2) The elicited KoD packet is likely to have poll value no larger than 10. (This follows because when ntpd servers send KoDs, the KoD's poll value $\tau_{\text {kod }}$ is at least as large as that in the query triggering the KoD. Meanwhile, the query triggering the KoD was a legitimate query from the client, and default maximum value of the poll in the client's query is 10.) The attacker can thus elicit a KoD that quiets the client for $\approx 2^{10}$ seconds (15 minutes), then elicit another KoD 15 minutes later, and continue this indefinitely.

Thus, patching clients to validate the KoD origin timestamp (CVE-2015-7704) does weaken, but does not eliminate, KoDrelated attacks.

\section{Low-rate off-path denial-of-service attack on NTP clients.}

It's tempting to argue that NTP clients are commonly preconfigured with many, and so KoD-related attacks on one server can be mitigated by the presence of the other servers. However, this is not the case. We now present a denial-ofservice attack that allows an off-path attacker to "turn off" NTP at a client by preventing the client from synchronizing to any of its preconfigured servers.

What are the implications of this attack? For the most part, the client will just sit there and rely on its own local clock for the time. If the client has accurate local time-keeping abilities, then this attack is unlikely to cause much damage. On the other hand, the client machine could be incapable of keeping time for itself, e.g., because it is in a virtual machine [69], or running CPU-intensive operations that induce clock drift. In this case, the client's clock will drift along, uncorrected by NTP, for the duration of attack.

The denial of service attack. The attack proceeds as follows:

1) The attacker sends a mode 3 NTP query to the victim client, and the client replies with a mode 4 NTP response. The attacker uses the reference ID in the mode 4 response to learn the IP of the server to which the client is synchronized.

2) The attacker spoofs/elicits a KoD packet with $\tau_{\text {kod }}$ from the server to which the client is synchronized. The client stops querying this server for at least $2^{\tau_{\text {kod }}} \mathrm{sec}$.

3) There are now two cases. Case 1: the client declines to take time from any its of other preconfigured servers; thus, the attacker has succeeded in deactivating NTP at the client. Case 2: The client will synchronize to one of its other preconfigured servers, and the attacker returns to step 1. To determine whether the client is in the Case 1 or Case 2, the attacker periodically sends mode 3 queries to the client, and checks if the reference ID in the mode 4 response has changed.

Thus, the attacker learns the IP addresses of all the preconfigured servers from which the client is willing to take time, and periodically (e.g., once every $2^{\tau_{\text {kod }}}$ seconds), spoofs/elicits KoD packets from each of them. The client will not synchronize to any of its servers, and NTP is deactivated.

Attack surface. For this attack to work, the client must (1) react to $\mathrm{KoD}$ packets by refraining from querying the KoD-sending server, (2) respond to NTP mode 3 queries with NTP mode 4 responses, and (3) be synchronized to an IPv4 NTP server. This creates a large attack surface: condition (1) holds for ntpd v4.2.6 and v4.2.8p3, the most recent reference implementation of NTP, and our scans (Section III-B) suggest that over 13M IPs satisfy condition (2).

\section{Recommendation: Kiss-o'-Death considered harmful.}

Following the disclosure of our work, many NTP implementations (including ntpd v4.2.8p4) have been patched to ensure that clients validate the origin timestamp on KoD packets (CVE-2015-7704), thus preventing our extremely lowrate off-path denial-of-service attack that uses spoofed KoD. However, our less severe priming-the-pump attack that allows the attacker to elicit a valid KoD (CVE-2015-7705) has not yet been addressed. Preventing this attack requires some rethinking of NTP's KoD and rate-limiting functionality.

One solution is to simply eliminate NTP's KoD and other rate-limiting functionality; this, however, eliminates a server's ability to deal with heavy volumes of NTP traffic. 
Alternatively, if clients are required to cryptographically authenticate their queries to the server, then it is no longer possible for an off-path attacker to prime the pump at the server by spoofing mode 3 queries from the client. Interestingly, however, a new proposal for securing NTP [63, Sec. 6.1.3] only suggests authenticating mode 4 responses from the server to client, but not mode 3 queries from client to server.

In the absence of authentication, another solution is to apply techniques developed for rate-limiting other protocols, e.g., Response Rate Limiting (RRL) in the DNS [67]. Like NTP, DNS is sent over unauthenticated UDP, and therefore is at risk for the same priming-the-pump attacks we discussed here. RRL addresses this by requiring a server to randomly respond to some fraction of the client's queries, even if that client is rate limited [68, Sec. 2.2.7]; thus, even a client that is subject to a priming-the-pump attack can still get some good information from the server. To apply this to NTP, a server that is rate-limiting a client with KoDs would send legitimate mode 4 responses (instead of a KoD) to the client's queries with some probability. For this to be effective, NTP clients should also limit the period for which they are willing to keep quiet upon receipt of a KoD; not querying the server for days $\left(\tau_{\text {kod }}=17\right)$ or even years $\left(\tau_{\text {kod }}=25\right)$ upon receipt of a single $\mathrm{KoD}$ packet is excessive and invites abuse.

\section{OfF-Path NTP Fragmentation AtTack}

We now show how an off-path attacker can hijack an unauthenticated NTP connection from a client to its server. The key ingredient in our attack is overlapping IPv4 packet fragments; therefore this attack succeeds a on small but nonnegligible set of clients and servers that use the IPv4 fragmentation policies described in Section VI-E. We will assume the client is preconfigured with only one server. (Some OSes (e.g., MAC OS X v10.9.5) actually do use this configuration, and in the full version [36] we show how to combine our KoD and reboot techniques to simulate this scenario for clients preconfigured with multiple servers.) We first explain why offpath attacks are challenging and give background on IPv4 packet fragmentation [50], [53], [55], [62]. Next, we present the attack itself, explain when it works, and conclude with a measurement study that sheds light on the number of clients and servers in the wild that are vulnerable to this attack.

\section{A. Why are off-path attacks hard?}

The goal of our attacker is to spoof a series of mode 4 response packets (Figure 11) from the server to the client. The spoofed response should contain bogus server timestamps (i.e., $T_{3}$ transmit timestamp, $T_{2}$ receive timestamp) in order to convince the client to accept bogus time from the server. This creates several hurdles for an off-path attacker who cannot see the communication between client and server:

First, there is the issue of nonces. Per Section V-A the attacker must spoof packets with the correct origin timestamp, which has about 32 bits of entropy. Our off-path attacker will not even try to learn the origin timestamp; instead, we use the origin timestamp from the honest mode 4 response from server to client, and use IPv4 packet fragmentation to overwrite other relevant fields of the NTP packet.
Second, since our attacker does not know NTP's origin timestamp, it cannot compute the UDP checksum. However, the UDP specification for IPv4 allows a host to accept any packet with UDP checksum of zero (which means: don't bother checking the checksum) [52, pg 2]. As such, our attacker uses IPv4 fragmentation to set the UDP checksum to zero.

Third, in order to convince the client's clock discipline algorithms to accept the attacker's bogus time, our attacker must spoof a stream of several packets that are acceptable to the clock discipline algorithm. To understand why, we note that for each valid mode 4 packet (or "sample") that an NTP client receives from its server, the client records the offset $\theta$ per equation (2) and delay $\delta$ per equation (1). The client keeps up to eight samples from each server, and selects the offset $\theta^{*}$ corresponding to the lowest delay $\delta^{*}$. It then computes the jitter $\psi$, which is the root-mean-square distance of the sample offsets from $\theta^{*}$, i.e.,

$$
\psi=\sqrt{\frac{1}{i-1} \sum_{i}\left(\theta_{i}-\theta^{*}\right)^{2}}
$$

(The sum is taken over the most recent samples.) The client only decides to update its local clock using the offset $\theta^{*}$ if the server's samples pass several complex tests posed by the clock discipline algorithm. One key test is TEST11:

TEST11. Check that the root distance $\Lambda$ does not exceed MAXDIST, a parameter that defaults to $1.5 \mathrm{sec}$. While RFC 5905 [42, Appendix A.5.5.2], ntpd v4.2.6 and ntpd v4.2.8 each use a slightly different definition of $\Lambda$, what matters is

$$
\Lambda \propto \psi+\left(\delta^{*}+\Delta\right) / 2+E+2^{\rho}
$$

where $\Delta$ is the root delay, $E$ is the root dispersion, and $\rho$ is the precision, all of which are read off the server's mode 4 packet (Figure 11). (Precision $\rho$ reveals the resolution of the server's local clock; e.g., $\rho=-12$ means the server's local clock is precise to within $2^{-12} \mathrm{sec}$ or $244 \mu \mathrm{s}$. Root delay $\Delta$ is the cumulative delay from the server to the 'root' (i.e., stratum 1 server) in the NTP client-server hierarchy from which the server is taking time; a stratum 1 server typically has $\Delta=0$. Root dispersion $E$ is an implementation-dependent quantity related to the $\Lambda$ value computed by the server.)

Our attacker must therefore ensure that his stream of packets passes TEST11, so that root distance $\Lambda<1.5$. To do this, the attacker uses IPv4 fragmentation to set several fields in the packet to tiny values, e.g., $\rho=-29, \Delta=0.002, E=0.003$ sec and stratum $=1$. Our attacker's main challenge is to ensure that jitter $\psi$ is small enough to pass TEST11. We show how this can be done in Section VI-D

\section{B. IPv4 packet fragmentation.}

Fragmentation is one of IP's original functionalities [53]; chopping a large packet into fragments that can be sent through networks that can only handle short packets. The length of the largest packet that a network element can accommodate is its 'maximum transmission unit (MTU)'. In practice, almost every network element today supports an MTU of at least 1492 bytes (the maximum payload size for Ethernet v2 [37, Sec. 7]). Back in 1981, however, RFC791 [53] required that "all hosts must be prepared to accept" IPv4 packets of length 576 bytes, while "every internet module must be able to forward" IPv4 packets 


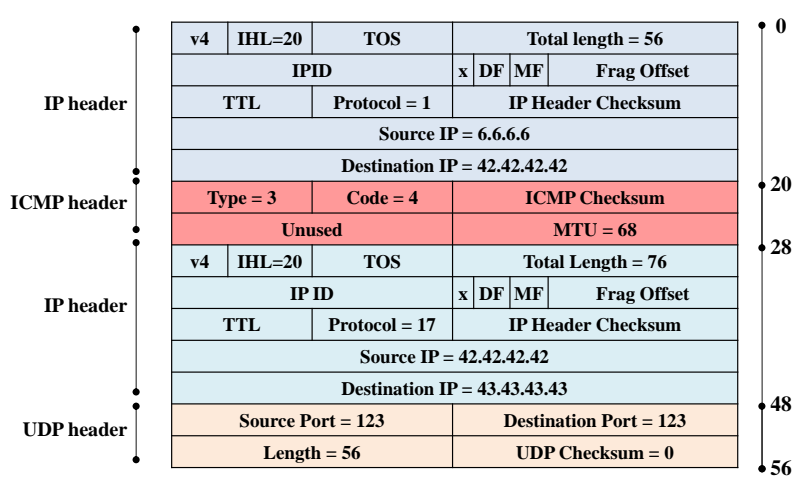

Fig. 3. ICMP Fragmentation Needed packet from attacker 6.6.6.6 telling server 42.42.42.42 to fragment NTP packets for client 43.43.43.43 to MTU of 68 bytes.

of length 68 bytes. The minimum IPv4 MTU for the Internet is therefore 68 bytes, but many OSes refuse to fragment packets to MTUs smaller than 576 bytes. Our attack only succeeds against servers that fragment to a 68 byte MTU; the attacker can therefore convince a server to chop an NTP packet into the two fragments on the right of Figure 4. Our measurements (Section VI-G) confirm that there are ten of thousands of NTP servers in the wild that do this.

ICMP Fragmentation Needed. How does a host learn that it needs to fragment packets to a specific MTU? Any network element on the path from sender to receiver can send a single ICMP fragmentation needed packet to the sender containing the desired MTU; this information is then cached for some OS-dependent period of time (e.g., 10 minutes by default on Linux 3.13.0 and MAC OS X 10.9.5). Figure 3 shows an ICMP fragmentation needed packet that signals to host 42.42.42.42 to fragment all NTP packets (UDP port 123) it sends to destination IP 43.43.43.43 to an MTU of 68 bytes. Since the host is not expected to know the IP addresses of all the network elements on its path, this packet can be sent from any source IP; in Figure 3 this source IP is 6.6.6.6. The payload of this ICMP packet contains an IP header and first eight bytes of a packet that has already been sent by host and exceeded the MTU [54]; for NTP, these eight bytes correspond to the UDP header. The sender uses this to determine which destination IP (i.e., 43.43.43.43) and protocol (i.e., UDP port 123) requires fragmentation. Our attacker (at IP 6.6.6.6) can easily send an ICMP fragmentation needed from off-path. Its only challenge is (1) choosing UDP checksum (which it sets to zero) and (2) matching the IPID in the ICMP payload with that in an NTP packet previously sent to the client (which it can do, see Section VI-D, and moreover some OSes don't bother checking this (e.g., Linux 3.13.0)).

IPv4 Fragmentation. How do we know that an IPv4 packet is a fragment? Fragment offset specifies the offset of a particular fragment relative to the beginning of the original unfragmented IP packet; the first fragment has an offset of zero. The more fragment $(M F)$ flag is set for every fragment except the last fragment. IPID indicates that a set of fragments all belong to the same original IP packet.

Fragment reassembly. How does a host reassemble a fragmented IPv4 packet? In the common case, the fragments are non-overlapping, so that the offset of one fragment begins immediately after the previous fragment ends. In this case, the host checks its fragment buffer for fragments with the same IPID, and pastes their payloads together according to their fragment offset, checking that the last fragment has a $\mathrm{MF}=0$ [53]. Fragment buffer implementations differ in different OSes [26], [30]. Meanwhile, the RFCs are mostly silent about reassembly of overlapping fragments, like the ones in Figure $44^{8}$ Several authors [6], [50], [55], [62] have observed that reassembly policies differ for different operating systems, and have undertaken to determine these policies using clever network measurement tricks. (Hilariously, wireshark has an overlapping fragment reassembly policy that is independent of its host OS [6] and is therefore useless for this purpose.) Our attacks also rely on overlapping fragments. Overlapping fragment reassembly policies are surprisingly complex, poorly documented, and have changed over time. Thus, instead of generically describing them, we just consider reassembly for the specific fragments used in our attack.

\section{Exploiting overlapping IPv4 fragments.}

Our attack proceeds as follows. The attacker sends the server a spoofed ICMP fragmentation needed packet (Figure 3 ) requesting fragmentation to a 68-byte MTU for all NTP packets sent to the client. If the server is willing to fragment to a 68-byte MTU, the server sends all of its mode 4 NTP responses as the two fragments on the right of Figure 4 Meanwhile, our attacker plants the two spoofed fragments on the left of Figure 4 in the client's fragment buffer. The spoofed fragments sit in the fragment buffer and wait for the server's legitimate fragments to arrive. The first spoofed fragment sets the UDP checksum to zero, and sets $\rho=-29, \Delta=0.002$, $E=0.003$ to tiny values so that the reassembled packet is more likely to pass TEST11. The second spoofed fragment sets the NTP receive timestamp $\left(T_{2}\right)$ and transmit timestamps $\left(T_{3}\right)$ to bogus time values. Both spoofed fragments must have the same IPID as the two legitimate fragments; we explain how to do this in Section VI-D. This process of planting spoofed fragments continues for every mode 4 NTP response that the server sends the client. Once the client has accepted the bogus time, the attacker spoofs KoDs (Section V-C) so the client stops updating its clock. (The attacker can check that the client accepted the bogus time by sending it a mode 3 query and checking the timestamps in the client's mode 4 response.)

The victim client receives the four overlapping fragments in Figure 4, in the order shown, with the leftmost fragment arriving earliest. How are they reassembled? One potential outcome is for the client to reject the fragments altogether because they are overlapping or too short. Otherwise, the first honest fragment arrives in the client's fragment buffer and is reassembled with one or both of the spoofed fragments, according to one of the reassembly outcomes shown in Figure 5 (Note: the second honest fragment will not reassemble with anything, because by the time it arrives in the buffer the earlier fragments have already reassembled.) In Outcome A the client prefers fragments that arrive earliest, pasting the first legitimate fragment underneath the two spoofed fragments that were waiting in the cache (i.e., the 'First' policy in the

\footnotetext{
${ }^{8}$ RFC $3128[41]$ does have some specific recommendations for overlapping IPv4 fragments in the case of TCP; NTP, however, is sent over UDP. Also, overlapping fragments are forbidden for IPv6.
} 


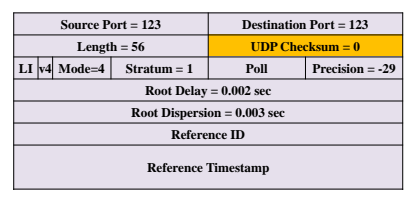

Receive Timestamp = June 3, 2020, 1:43:03 PM UTC

Transmit Timestamp = June 3, 2020, 1:44:55 PM UTC
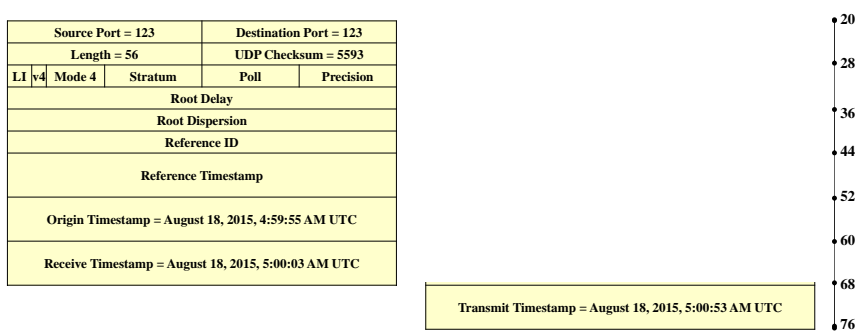

Fig. 4. IPv4 fragments for our attack: $1^{\text {st }}$ and $2^{\text {nd }}$ spoofed fragments followed by $1^{\text {st }}$ and $2^{\text {nd }}$ legitimate fragments.
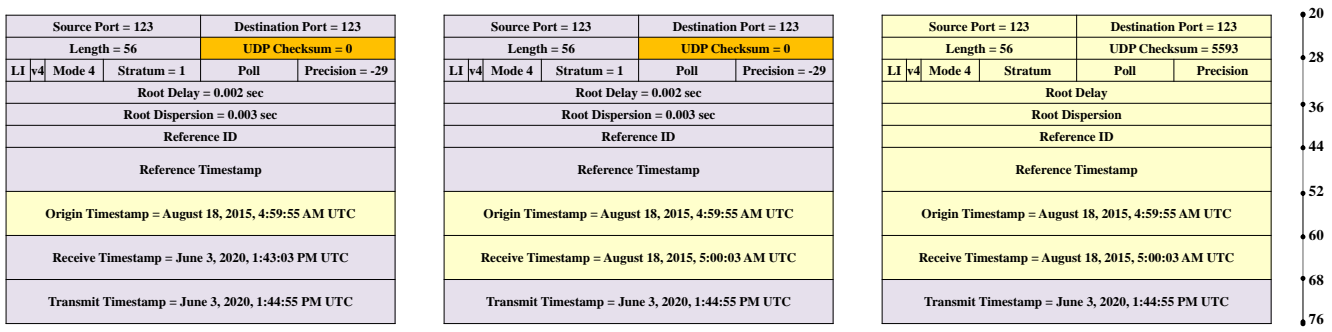

Fig. 5. Different ways our fragments may be reassembled. From left to right: Outcome A, Outcome B, Outcome C.

Paxson/Shankar overlapping-packet reassembly model [62]). In Outcome B, the client prefers an earlier fragment with an offset that is less than or equal to a subsequent fragment (i.e., the 'BSD' policy of [50], [62]). In Outcome C the client prefers fragments that arrive later over those that arrive earlier.

In which outcome does our attack succeed? In Outcome C, the packet is dropped due to its incorrect UDP checksum, and our attack fails. In Outcomes A and B, our off-path attacker successfully injects packets with the correct origin timestamp and UDP checksum. However, in Outcome B, the attacker controls only the transmit timestamp $T_{3}$ in the reassembled packet. Because passing TEST11 (equation (4)) constrains delay $\delta$ to be $<1 \mathrm{sec}$ (equation (11) it follows that the spoofed $T_{3}$ must be within $1 \mathrm{sec}$ of the legitimate receive timestamp $T_{2}$, making our attack much less interesting. Our attack therefore works best in Outcome A, where the attacker controls both the $T_{3}$ and $T_{2}$; by setting $T_{2} \approx T_{3}$, the delay $\delta$ is small enough to pass TEST11, even if the spoofed $T_{2}$ and $T_{3}$ are very far from the legitimate time.

\section{Planting spoofed fragments in the fragment buffer.}

Because a client will only take time from a server that provides several self-consistent time samples (Section VI-A), our attacker must craft a stream of NTP mode 4 responses. In achieving this, our attacker must surmount two key hurdles:

Hurdle 1: Jitter. Passing TEST11 also constrains the jitter $\psi$ to be $<1 \mathrm{sec}$ (equation (3)); to achieve this, the offset values $\theta$ in the reassembled stream of packets must be consistent to within about $1 \mathrm{sec}$. Why is this difficult? The key problem is that the offset $\theta$ is determined by the timestamps $T_{2}$ and $T_{3}$ set in the attacker's second spoofed fragment (Figure 4), as well as the origin and destination timestamps $T_{1}, T_{4} . T_{1}$ corresponds to the moment when the legitimate client sends its query, and is unknown to our off-path attacker. Moreover, $T_{1}$ roughly determines $T_{4}$, which roughly corresponds to the moment when the first legitimate fragment reassembles with the spoofed fragments in the client's fragment buffer. Now suppose the fragment buffer caches for $30 \mathrm{sec}$. This means that timestamps $T_{2}$ and $T_{3}$ (from attacker's second spoofed fragment) can sit in the fragment buffer for anywhere from 0 to $30 \mathrm{sec}$ before reassembly at time $T_{4}$ (Figure 4). Thus, the offset $\theta$ in the reassembled packet can vary from 0 to $30 \mathrm{sec}$, so that jitter $\psi \approx 30 \mathrm{sec}$ and the attack fails TEST11.

Hurdle 2: IPID. Our attacker must ensure that the IPID of the spoofed fragments planted in the fragment buffer (the left two fragments in Figure 47 match the IPID of the legitimate fragments sent by the server (the right two fragments in Figure 47; this way, the spoofed fragments will properly reassemble with the legitimate fragments.

Surmounting these hurdles. To surmount the first hurdle, our attacker ensures that the client's fragment buffer always contains fresh copies of the second spoofed fragment that are no more than $1 \mathrm{sec}$ old. Suppose that the client's fragment cache is a FIFO queue that holds a maximum of $n$ fragments. (Windows XP has $n=100$ [30], and the Linux kernel in [26] has $n=64$ ). Then, every second, the attacker sends the client $n / 2$ copies of its first spoofed fragment (each with different IPIDs), and $n / 2$ copies of the second spoofed fragment, per Figure 4 Each second spoofed fragment has (1) IPID corresponding to one of the first spoofed fragments, and (2) timestamps $T_{2}$ and $T_{3}$ corresponding to the (legitimate) time that the fragment was sent plus some constant value (e.g., $x=+10$ mins, where $x$ represents how far the attacker wants to shift the client forward/backward in time). Thus, every second, a fresh batch of $n$ fragments evicts the old batch of $n$ fragments. The reassembled packets have offset within $\approx 1$ sec, so that jitter $\psi \approx 1 \mathrm{sec}$, and the attacker passes TEST11.

To surmount the second hurdle, our attack exploits the fact that IPIDs are often predictable. Several policies for setting IPID exist in the wild, including: globally-incrementing, i.e., the OS increments IPID by one for every sent packet, perdestination-incrementing, i.e., the OS increments IPID by one every packet sent to a particular destination IP, and random, i.e., the IPID is selected at random for every packet [20]. Random IPIDs thwart our attacks. However, when the server uses an incrementing IPID policy, the following techniques 
allow our attacker to plant several copies of the spoofed fragments with plausible IPIDs (cf., [20], [26], [30]):

Globally incrementing IPIDs: Before sending the client the $n / 2$ copies of the spoofed fragments, our attacker pings the server to learn its IPID $i$, and sets IPID of its spoofed packets accordingly (i.e., to $i+1, i+2, \ldots, i+n / 2$ ).

Per-destination incrementing IPIDs: Gilad and Herzberg [20] [30] show how per-destination incrementing IPIDs can be inferred by a puppet (adversarial applet/script that runs in a sandbox) on the client or server's machine, while Knockell and Crandall [30] show how to do this without puppets. Thus, at the start of our attack, our attacker can use [20], [30]'s techniques to learn the initial IPID $i$, then uses $i$ to set IPIDs on the $n / 2$ copies of its pairs of spoofed fragments. The choice of IPIDs depends on the polling interval, i.e., the frequency at which the client queries the server. NTP default poll values range from $\tau=6\left(2^{6}=64 \mathrm{sec}\right)$ to $\tau=10$ (1024 seconds) [42]. If the attacker knew that the client was polling every 64 seconds, it could send $n / 2$ copies of the spoofed fragments with IPID $i+1, \ldots, i+n / 2$, and then increment $i$ every 64 seconds.

More commonly, however, the polling interval is unknown. To deal with this, the attacker can predict the IPID under the assumption that the client and server consistently used the minimum (resp., maximum) polling interval, and ensures that all possible IPIDs in between are planted in the buffer. As an example, suppose that 2048 seconds (30 mins) have elapsed since the attacker learned that the IPID is $i$. At one extreme, the client and server could have consistently used the minimum default polling interval of $2^{\tau}=64 \mathrm{sec}$; thus, $i_{\max }=$ $i+2048 / 64=i+32$. At the other extreme, the client and server could have consistently used the maximum default polling interval of $2^{\tau}=1024 \mathrm{sec}$; then $i_{\min }=i+2048 / 1024=i+2$. Then, the attacker must send pairs of spoofed fragments with IPIDs ranging from $i_{\min }=i+2$ to $i_{\max }=i+32$. This works as long as the fragment buffer can hold $i_{\max }-i_{\min }>30 \cdot 2$ fragments (as in e.g., Linux [26] and Windows XP [30]). When $2\left(i_{\min }-i_{\max }\right)$ exceeds the size of the fragment buffer $n$, the attacker repeats [20], [30]'s techniques to learn IPID again.

\section{E. Conditions required for our attack.}

In summary, our attack succeeds for a given victim NTP server and victim NTP client if the following hold:

1) the server accepts and acts on ICMP fragmentation needed packets for a 68-byte MTU, and

2) the server uses globally-incrementing or per-destinationincrementing IPID, and

3) the client reassembles overlapping IPv4 fragments as in Outcome A of Figure 5 .

\section{F. Proof-of-concept implementation of our attack.}

We implemented a proof of concept of our attack on three lab machines. Our server had per-destination incrementing IPID. We perform the small-step-big-step attack (Section IV-B) from off path against an ntpd v4.2.6p5 client, with a 'small step' of 10 minutes, and a 'big step' of 1 day.

Server. Our victim server ran ntpd v4.2.8p2 on Linux 3.13.024-generic kernel which uses a per-destination incrementing IPID. This Linux kernel has configuration parameter min_pmtu that determines the minimum MTU to which the OS is willing to fragment packets upon receipt of an ICMP fragmentation needed packet; we manually set min_pmtu to 68 , so that the server would satisfy the first condition of our attack ${ }^{9}$

Client. Choosing the right client was a challenge. It is extremely difficult to find documentation on overlapping fragment reassembly policies for popular OSes. After testing various OSes, we tried using the Snort IDS to emulate a network topology where a middlebox reassembles fragmented packets before passing them to endhosts [10]. Unfortunately, Snort's frag3 engine, which reassembles overlapping IPv4 fragments according to various policies, exhibited buggy behavior with UDP (even though it worked fine with the ICMP fragments used in [50]). Finally, we gave up and wrote our own fragment buffer in python and scapy, running it on a Linux 3.16.0-23generic OS with ntpd v4.2.6p5. The fragment buffer itself is a FIFO queue with capacity $n=28$ fragments and timeout $t=30 \mathrm{sec}$. Fragments older than $t$ are evicted from the queue. When the queue is full, a newly-arrived fragment evicts the oldest fragment. The buffer reassembles packets according to the 'First' policy ${ }^{10}$ in [62] (i.e., Outcome A in Figure 4).

Attacker. Our attacker machine ran code written in scapy. Before the attack starts, we let the NTP client synchronize to the server. After that, our attacker machine should infer the IPID the server uses to send mode 4 packets to the client; rather than reimplementing the IPID-inference techniques of [20], [30], we just have the client machine send the initial IPID $i$ directly to the attack machine. At this point, the client no longer reveals any more information to the attacker, and the attack starts. The attacker first sends the server a spoofed ICMP fragmentation needed packet requesting fragmentation to a 68byte MTU for the client; the server caches the request for 10 minutes, and starts sending the two rightmost fragments in Figure 4 To keep the server fragmenting, the attacker sends a fresh ICMP fragmentation needed every 10 minutes.

Per Section VI-D, each second our attacker needs to send the client a fresh batch of $n / 2$ pairs of the two leftmost fragments in Figure 4. Each pair had IPID in $\{(i+1), \ldots,(i+$ $n / 2-1)\}$, with $i$ incremented every 70 seconds ${ }^{11}$ Our attack machine was a fairly lame eight-year old Fujitsu x86_64 with 1.8GB of memory running Linux 3.16.0-24-generic, and thus could only manage to send thirteen pairs of the required fragments within 1 second. We therefore set the size of the FIFO queue on our client machine to $n=28$. Our attacker uses the fragmentation attack to launch a "small-step-big-step" attack : First, it sets receive and transmit timestamps $T_{2}$ and $T_{3}$ in its second spoofed fragment to shift the client 10 minutes back in time. Once the client enters 'STEP' mode, it sets $T_{2}$ and $T_{3}$ to shift the client one day back in time. (Note that an

\footnotetext{
${ }^{9}$ The default value for min_pmtu in Linux exceeds 500 bytes $[59 \mid$, so that the vast majority of NTP servers running on Linux should not be vulnerable to our attack. (Linux 2.2.13 is one notable exception; see Section VI-G) However, our measurements in Section VI-G indicate that servers in the wild do fragment to a 68-byte MTU; we just use Linux 3.13.0 as a proof-of-concept in our lab.

${ }^{10}$ The 'First' policy of 62 requires reassembly to prefer the fragment that was received earliest by fragment buffer.

${ }^{11}$ NTP uses a randomized algorithm to set the polling interval. Our client had not been running for long, so its polling interval was $\tau=6$ (64 sec), which translates to intervals randomly chosen from the discrete set $\{64,65,66,67\}$ sec. We therefore increment $i$ every 70 seconds.
} 


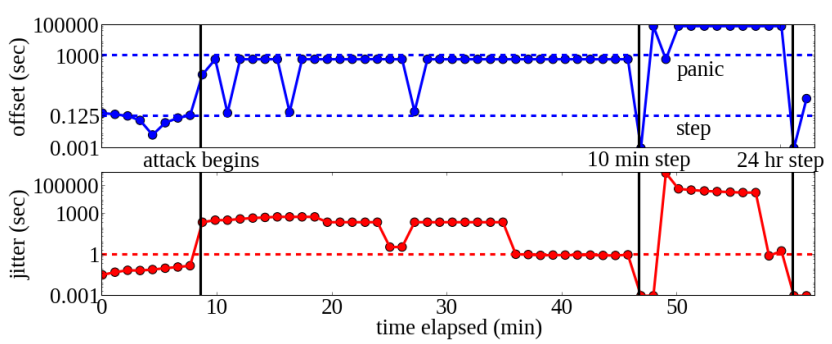

Fig. 6. Absolute value of offset $\theta$ (above) and jitter $\psi$ (below) computed by the client during a proof-of-concept implementation of our attack.

off-path attacker can check that the client is in 'STEP' mode by querying the client and checking for 'STEP' in the reference ID of the response [42, Fig. 13].)

Results (Figure 6). We plot the results of one run of our attack, obtained from the client's ntpq program. We plot offset $\theta$ (equation (2) ) computed by the client for each mode 4 packet the client (thinks it) received from the server. The horizontal lines on the offset plot represent NTP's default panic threshold $(1000 \mathrm{sec})$ and 'STEP' threshold (125 ms). We also plot jitter $\psi$ (equation (3) ) computed by the client from its eight most recent offset samples. Recall that the client will only synchronize to the server if $\psi<1 \mathrm{sec}$ (VI-D). Before the attack begins, the client and server are synchronized and offset is small. Once the attack begins, offset jumps to about 600 seconds (10 minutes). Figure 6 also shows some spikes where the offset jumps back down to a few msec. These spikes occur during cache misses, when our attacker fails to plant fragments with the right IPID in the fragment buffer; this allows the two legitimate fragments to reassemble so that the client gets sample of the correct time. The attacker pays a price each time a cache miss causes an inconsistency in the offset values; for example, at time 25 mins, the attacker crafts enough packets to force the jitter to about $10 \mathrm{sec}$, but two samples later it suffers a cache miss, causing jitter to jump up again. Eventually, the attacker crafts enough packets to keep jitter below $1 \mathrm{sec}$ for some period of time, and the client accepts the time, enters 'STEP' mode, and clears its state. Once in 'STEP' mode, the attacker manages to craft nine consecutive packets, causing jitter to drop below 1 sec and sending the client back in time for another 24 hours.

\section{G. Measuring the attack surface: Servers.}

How often are the conditions required for our attack (Section VI-E) satisfied in the wild? We answer this question by scanning our dataset of 13M NTP servers (Section III-B) to find servers satisfying the two conditions for our attack per Section VI-E (1) fragmenting to a 68-byte MTU, and (2) using incrementing IPID. To avoid harming live NTP servers with this scan, we send only ICMP packets or mode 3 NTP queries (which do not set time on the server).

Fragmenting to 68-byte MTU. We send each server in our list (1) an NTP mode 3 query and capture the corresponding NTP mode 4 response, and then (2) send an ICMP fragmentation needed packet requesting fragmentation to a 68-bytes for NTP packets sent to our measurement machine (as per the packet in Figure 3, where UDP checksum is zero and IPID inside the ICMP payload is that in the captured mode 4 NTP response), and finally (3) send another NTP mode 3 query. If the server fragments the final mode 4 NTP response it sends us, we conclude it satisfies condition (1) for our attack.
TABLE VI. IPID BEHAVIOR OF NON-BAD-TIMEKEEPERS SATISFYING CONDITIONS (1), (2) OF SECTIONVI-E

\begin{tabular}{|c||c|c|c|c|c|c|c|}
\hline IPID & Per-Dest & \multicolumn{6}{|c|}{ Globally incrementing } \\
behavior & $\Gamma=1$ & $\Gamma=10$ & $\Gamma=25$ & $\Gamma=50$ & $\Gamma=100$ & $\Gamma=250$ & $\Gamma=500$ \\
\hline \# servers & 2,782 & 5,179 & 2,691 & 533 & 427 & 135 & 55 \\
\hline
\end{tabular}

Server IPID behavior. Next, we check the IPID behavior of each server that was willing to fragment to a 68-byte MTU. To do this, we send each IP five different NTP mode 3 queries, interleaving queries so that at about $30 \mathrm{sec}$ elapse between each query to an IP. We then check the IPIDs for the mode 4 responses sent by each IP. If IPID incremented by one with each query, we conclude the server is vulnerable because it uses a per-destination-incrementing IPID. Otherwise, we determine the gap between subsequent IPIDs; if all gaps were less than a threshold $\Gamma$ (for $\Gamma=\{10,25,100,250,500\}$ ), we conclude that the server uses a globally-incrementing IPID.

Results of server scan. Out of the $13 \mathrm{M}$ servers we scanned, about $24 \mathrm{~K}$ servers were willing to fragment to a 68-byte MTU. $10 \mathrm{~K}$ of these servers have bigger problems than just being vulnerable to our attacks: they were either unsynchronized (i.e., either stratum 0 or stratum 16) or bad timekeepers (i.e., with offset $\theta>10 \mathrm{sec}$ ). However, we did find 13,081 'functional' servers that fragment to a 68-byte MTU. As shown in Table VI. the vast majority $(11,802$ servers $)$ of these are vulnerable to our attack because they use an incrementing IPIDs that grow slowly within a 30-second window. In fact, most use a globally-incrementing IPID, which is easier to attack than a per-destination IPID (see Section VI-D).

Who are these vulnerable servers? The majority $87 \%$ $(10,292$ servers) are at stratum 3 , but we do see 14 vulnerable stratum 1 servers and 660 vulnerable servers with stratum 2. Indexing these with our (very incomplete) topology data, we find that 11 servers are at the root of subtrees with over 1000 clients, and 23 servers have over 100 clients each. One vulnerable stratum 2 server, for example, is in South Korea and serves over $19 \mathrm{~K}$ clients, another with over $10 \mathrm{~K}$ clients is in a national provider in the UK, one with over $2 \mathrm{~K}$ clients serves a research institute in Southern Europe, and two with over 7K clients are inside a Tier 1 ISP. Cross-referencing these servers to our May $2015 r v$ data, we find that the vast majority $(9,571$ out of the 11,803 vulnerable servers) are running Linux 2.2.13; the other significant group is 1,295 servers running "SunOS".

\section{H. Measuring the attack surface: Clients.}

Determining how many clients in the wild satisfy the third condition of our attack (Section VI-E) was significantly more complex. To measure how an NTP client reassembles overlapping IPv4 fragments, we can use [50], [62]'s technique of sending fragmented pings. To check for reassembly per Outcome A in Figure 5, we send four ping fragments with offsets corresponding exactly to those in Figure 4 If the client reassembles them as in Outcome A, the reassembled ping will have a correct ICMP checksum and elicit a ping response from the client; otherwise, the client will ignore them. We repeat this with four other ping fragments to check for Outcome B.

Before we could deploy this technique in the wild, we hit an important complication: Teardrop [9], an ancient implementation bug (from 1997) with devastating consequences. 
In a teardrop attack, the attacker sends two overlapping IPv4 fragments to an OS, and the OS crashes. Most OSes were patched for this over fifteen years ago, but some firewalls and middleboxes still alarm or drop packets when they see overlapping IPv4 fragments. Worse yet, legacy operating systems may not be patched, and new IP stacks might have reintroduced the bug. This is a big problem for us: this measurement technique inherently requires overlapping IPv4 fragments, and thus inherently contains a teardrop attack. We therefore cannot run this measurement on all 13M NTP clients we found in the wild, since we don't know what OSes they might be running. Instead, we deal with this in two ways.

First, we have developed a website for users to measure the vulnerability of their NTP clients, i.e., whether they reassemble packets as in Outcome A. (http://www.cs.bu.edu/ goldbe/ NTPattack.html) To prevent the site from becoming a teardrop attack vector, we require users running the measurement to be on the same IP prefix as the measured NTP client.

Second, we can send our measurements to NTP clients that we know are patched for Teardrop. Teardrop affects version of Linux previous to 2.0.32 and 2.1.63 [9]; thus, we can use the $r v$ data from the openNTPproject to determine which clients are running on patched Linux versions, and send our measurements to those clients only. We did this for 384 clients that responded to $r v$ queries with "Linux/3.8.13", a kernel released in May 2013, well after Teardrop was patched. Five clients responded with pings reconstructed as in Outcome A, 51 clients with pings reconstructed as in Outcome B.

This is interesting for two reasons. Most obviously, this gives evidence for the presence of reassembly per Outcome $A$ in the wild, which means that there are NTP clients that are vulnerable to our attack. But it also suggests that this fragmentation reassembly is not always done by the endhost itself; if it had, all 384 servers would have responded in the same way. Thus, we speculate that these five clients responded to our ping because they were sitting behind a middlebox that performs fragment reassembly for them [10].

\section{Recommendations: Fragmentation still considered harmful.}

Our measurements suggest that the attack surface for our NTP fragmentation attack is small but non-negligible. Thousands of NTP servers satisfy the conditions required by our attack (Section VI-E). However, our attack only succeeds if the victim client is synchronized to a vulnerable server, and reassembles fragmented packets according to the third condition required for our attack (Section VI-E). Unfortunately, we could not safely measure which NTP clients reassemble packets in this way, although we do find evidence of vulnerable clients.

Perhaps the simplest way to protect the NTP ecosystem is to ensure that any OS running an NTP server does not fragment packets to a 68 byte MTU; indeed, many OSes already do this (e.g., Linux [59], Windows [40]). On the client side, the OS should drop overlapping NTP fragments, as should middleboxes that reassemble IPv4 fragments like [10].

Source port randomization? One might wonder whether our attack is thwarted by UDP source-port randomization. Unfortunately, the answer is no. Suppose conditions (1), and (2) of Section VI-E hold, and modify our attack as follows: replace the first spoofed fragment on the left of Figure 4 with an identical fragment that has its UDP source port and destination port fields sliced off. (The replacement fragment has fragment offset of 24 bytes.) Then, if the client reassembles the packet according to the 'First' policy of [62], then the packet will look just like the one reassembled per Outcome A in Figure 5, except with the legitimate UDP source/dest ports, and the attack will succeed.

\section{RELATED WORK}

NTP security. While NTP-based DDoS amplification attacks have generated widespread interest (see e.g., [14]), there is less research [13], [29], [43], [45], [60] on the implications of shifting time via NTP attacks. A few researchers [43], [45], [60], [61] have considered the implications of attacks on NTP traffic, and [60], [61] demonstrated on-path attacks on timing clients that update their clocks at predictable intervals. We consider on-path attacks on the full NTP implementation (ntpd), which does not perform clock updates in a predictable fashion, and present new off-path attacks. Complementary to our work are efforts to identify software bugs in ntpd [49], [57], including several that were made public at the same time as our work [11]; because ntpd typically operates as root on the host machine, we expect that interest in this area will only continue to increase. Our work is also related to older NTP measurement studies [44], [47], as well as the recent work of [14]; [14] studies NTP's use for DDoS amplification, but we focus on the integrity of timing information. Finally, concurrent to our work is a study that measures network latency using NTP [17].

IPv4 Fragmentation. Our work is also related to research on exploiting IPv4 packet fragmentation for e.g., off-path attacks on operating systems [9], DNS resolvers [26], TCP connections [20], [22], and to evade intrusion detection systems [6], [50], [55], [62] and exploit side channels [30]. Unlike most prior work, however, we had to use fragmentation to craft a stream of self-consistent packets, rather than a single packet. Our attack also exploits problems with overlapping IPv4 fragments [6], [50], [62] and tiny IPv4 fragments, and should provide some extra motivation for OSes/middleboxes to drop tiny/overlapping fragments, rather than reassemble them.

\section{CONCLUSION}

Our results suggest four ways to harden NTP:

1) In Section IV we discuss why freshly-restarted ntpd clients running with the $-g$ configuration (which is the default installation for many OSes) are vulnerable to quick time shifts of months or years. We also present a 'small-step-big-step' attack, captured in CVE-2015-5300, that allows an on-path attacker to stealthily shift time on a freshly-restarted ntpd client. Different versions of the small-step-big-step attack succeed on ntpd v4.2.6 and ntpd v4.2.8. To protect against these attacks, users can either stop using the $-g$ configuration, or monitor their systems for suspicious reboots of the OS or of ntpd. Section IV-C also has recommendations for implementors who wish to patch against our small-step-big-step attack.

2) We showed how NTP's rate-limiting mechanism, the Kiss-o-Death packet $(\mathrm{KoD})$, can be exploited for off-path denial-of-service attacks on NTP clients (Section V-C). We find that ntpd versions earlier than v4.2.8p3 allow for trivial 
spoofing of KoD packets, and that this can be exploited to disable NTP on clients in the Internet using only a single attacking machine. The KoD-spoofing vulnerability (CVE2015-7704) has been patched in ntpd 4.2.8p4, following the disclosure of our work. This patch, however, does not prevent off-path attackers from eliciting valid KoDs from the server via a priming-the-pump technique (CVE-2015-7705); the primingthe-pump technique, however, does require the attacker to expend more resources (i.e., send more packets). Therefore, we argue in Section V-D that NTP should either (1) eliminate its KoD functionality, (2) require NTP clients to cryptographically authenticate their queries to NTP servers, or (3) adopt more robust rate limiting techniques, like [67].

3) In Section VI we showed how an off-path attacker can use IPv4 fragmentation to hijack an NTP connection from client to server. Because our attack requires server and client to run on operating systems that use less-common IPv4 fragmentation policies, we have used a measurement study to quantify its attack surface, and found it to be small but non-negligible. As we argue in Section VI-I, NTP servers should run on OSes that use a default minimum MTU of $\approx 500$ bytes, as in recent versions of Linux and Windows [40], [59]. OSes and middleboxes should also drop overlapping IPv4 fragments. We have also set up a website where operators can test their NTP clients for vulnerability to our fragmentation attacks. ${ }^{12}$

4) Each of our attacks has leveraged information leaked by the reference ID field in the NTP packet (Figure 1). (In Section IV] we use the reference ID to learn that the client was in the 'INIT' or 'STEP' state. In Section V-C our offpath attacker used the reference ID to learn the IPs of client's servers.) Moreover, since the purpose of the reference ID is to prevent timing loops (where A takes time from B who takes time from A [65]) any extra information leaked in the reference ID should be limited. One idea is to use a salted hash of the server's IP; see the full version [36] for more details.

5) Our attacks also exploited the fact that, by default, ntpd sends mode 4 responses in response to any mode 3 query sent by any IP in the Internet. Thus, it makes sense to limit the set of IPs that can communicate with an NTP client, especially for clients that are not designed to serve time to the Internet at large. (My laptop, for example, should not be responding to NTP queries from anyone.) An organization can also run its entire NTP infrastructure (including stratum 1 servers) behind a firewall, to avoid attacks from the public Internet.

Our work also motivates taking another look at cryptographic authentication for NTP [23], [45], [56], [63].

\section{ACKNOWLEDGEMENTS}

We thank the Network Time Foundation, NTPsec, the RedHat security team and Cisco for promptly issuing patches and working with vendors to mitigate our attacks.

We are especially grateful to Jared Mauch for giving us access to the openNTPproject's data and servers and for helping us coordinate the disclosure of this work, and to Miroslav Lichvar for his careful review of our work, especially the small-step-big-step attack. We thank Quinn Shamblin, Lora Fulton, Paul Stauffer and Joe Szep for IT and research

\footnotetext{
${ }^{12}$ http://www.cs.bu.edu/ goldbe/NTPattack.html
}

support, Haya Shulman and Yossi Gilad for discussions about packet fragmentation, Nadia Heninger for discussions about TLS, Dimitris Papadopoulos for discussions about DNS, Ethan Heilman for discussions about the RPKI, Leonid Reyzin for general wisdom, and the anonymous NDSS reviewers for comments on this manuscript. We had productive discussions about NTP security with Majdi Abbas, Daniel Franke, Sue Graves, Matt Van Gundy, Graham Holmes, David Mills, Danny Mayer, Hai Li, Karen ODonoghue, Clay Seaman-Kossmeyer, Harlan Stenn, Eric S. Raymond, Amar Takar, Dave Ward, and Florian Weimer. This research was supported, in part, by NSF awards 1347525, 1414119 and 1012910 and a gift from Cisco.

\section{REFERENCES}

[1] Autokey Configuration for NTP stable releases. The NTP Public Services Project: http://support.ntp.org/bin/view/Support/ ConfiguringAutokey (Accessed: July 2015).

[2] DoD Customers : Authenticated NTP. http://www.usno.navy.mil// USNO/time/ntp/dod-customers (Accessed: July 2015).

[3] The NIST authenticated ntp service. http://www.nist.gov/pml/div688/ grp40/auth-ntp.cfm (Accessed: July 2015), 2010.

[4] Amazon. Simple storage service (s3): Signing and authenticating rest requests. http://docs.aws.amazon.com/AmazonS3/latest/dev/ RESTAuthentication.html (Accessed Aug 2015).

[5] Axel K. ntpd access restrictions, section 6.5.1.1.3. allow queries? http: //support.ntp.org/bin/view/Support/AccessRestrictions. Feb. 2015.

[6] M. Baggett. IP fragment reassembly with scapy. SANS Institute InfoSec Reading Room, 2012.

[7] E. Barker and A. Roginsky. Transitions: Recommendation for transitioning the use of cryptographic algorithms and key lengths. NIST Special Publication. http://csrc.nist.gov/publications/nistpubs/800-131A/ sp800-131A.pdf 800:131A, 2011.

[8] L. Bicknell. NTP issues today. Outages Mailing List http: //mailman.nanog.org/pipermail/nanog/2012-November/053449.html November 2012.

[9] BUGTRAQ mailing list. Linux and Windows IP fragmentation (Teadrop) bug, 1997. http://insecure.org/sploits/linux.fragmentation.teardrop. html

[10] Checkpoint. IP fragments (UTM-1 appliance). https://www.checkpoint. $\mathrm{com} / \mathrm{smb} / \mathrm{help} / \mathrm{utm} 1 / 8.1 / 2032 . \mathrm{htm}$

[11] A. Chiu. Cisco identifies multiple vulnerabilities in network time protocol daemon (ntpd). TALOS Blog http://blog.talosintel.com/2015/ 10/ntpd-vulnerabilities.html October 2015.

[12] D. Cooper, E. Heilman, K. Brogle, L. Reyzin, and S. Goldberg. On the risk of misbehaving RPKI authorities. HotNets XII, 2013.

[13] corbixgwelt. Timejacking \& bitcoin: The global time agreement puzzle (culubas blog), 2011. http://culubas.blogspot.com/2011/05/ timejacking-bitcoin_802.html (Accessed Aug 2015).

[14] J. Czyz, M. Kallitsis, M. Gharaibeh, C. Papadopoulos, M. Bailey, and M. Karir. Taming the 800 pound gorilla: The rise and decline of NTP DDoS attacks. In Proceedings of the 2014 Internet Measurement Conference, pages 435-448. ACM, 2014

[15] M. d'Itri. Hacking team and a case of bgp hijacking. http://blog.bofh. it/id_456 July 2015.

[16] DropBox. Core API. https://www.dropbox.com/developers/core/docs (Accessed Aug 2015)

[17] R. Durairajan, S. K. Mani, J. Sommers, and P. Barford. Time's forgotten: Using ntp to understand internet latency. HotNets'15, November 2015.

[18] Z. Durumeric, E. Wustrow, and J. A. Halderman. Zmap: Fast internetwide scanning and its security applications. In USENIX Security, pages 605-620. Citeseer, 2013.

[19] P. Eckersley and J. Burns. An observatory for the SSLiverse. DEFCON'18, July 2010.

[20] Y. Gilad and A. Herzberg. Fragmentation considered vulnerable. ACM Trans. Inf. Syst. Secur, 15(4), 2013 
[21] S. Goldberg. Why is it taking so long to secure internet routing? Communications of the ACM: ACM Queue, 57(10):56-63, 2014.

[22] F. Gont. RFC 5927: ICMP attacks on TCP. Internet Engineering Task Force (IETF), 2010. https://tools.ietf.org/html/rfc5927

[23] B. Haberman and D. Mills. RFC 5906: Network Time Protocol Version 4: Autokey Specification. Internet Engineering Task Force (IETF), 2010. https://tools.ietf.org/html/rfc5906

[24] E. Hammer-Lahav. RFC 5849: The OAuth 1.0 Protocol. Internet Engineering Task Force (IETF), 2010. https://tools.ietf.org/html/rfc5849

[25] E. Heilman, D. Cooper, L. Reyzin, and S. Goldberg. From the consent of the routed: Improving the transparency of the RPKI. ACM SIGCOMM'14, 2014.

[26] A. Herzberg and H. Shulman. Fragmentation considered poisonous, or: One-domain-to-rule-them-all. org. In Communications and Network Security (CNS), 2013 IEEE Conference on, pages 224-232. IEEE, 2013.

[27] D. Kaminsky. Black ops 2008: It's the end of the cache as we know it. Black Hat USA, 2008.

[28] K. Kiyawat. Do web browsers obey best practices when validating digital certificates? Master's thesis, Northeastern University, 2014.

[29] J. Klein. Becoming a time lord - implications of attacking time sources. Shmoocon Firetalks 2013: https://youtu.be/XogpQ-iA6Lw 2013.

[30] J. Knockel and J. R. Crandall. Counting packets sent between arbitrary internet hosts. In 4th USENIX Workshop on Free and Open Communications on the Internet (FOCI'14), 2014.

[31] B. Knowles. NTP support web: Section 5.3.3. upstream time server quantity, 2004. http://support.ntp.org/bin/view/Support/ SelectingOffsiteNTPServers\#Section_5.3.3. (Accessed July 2015).

[32] J. Kohl and C. Neuman. RFC 1510: The Kerberos Network Authentication Service (V5). Internet Engineering Task Force (IETF), 1993. https://tools.ietf.org/html/rfc1510

[33] O. Kolkman, W. Mekking, and R. Gieben. RFC 6781: DNSSEC Operational Practices, Version 2. Internet Engineering Task Force (IETF), 2012. http://tools.ietf.org/html/rfc6781

[34] A. Langley. Revocation still doesn't work. https://www.imperialviolet. org/2011/03/18/revocation.html April 292014.

[35] M. Lepinski and S. Kent. RFC 6480: An Infrastructure to Support Secure Internet Routing. Internet Engineering Task Force (IETF), 2012. https://tools.ietf.org/html/rfc6480

[36] A. Malhotra, I. E. Cohen, E. Brakke, and S. Goldberg. Attacking the network time protocol. ePrint Cryptology Archive (Report 2015/1020) https://eprint.iacr.org/2015/1020.pdf 2015.

[37] L. Mamakos, K. Lidl, J. Evarts, D. Carrel, D. Simone, and R. Wheeler. RFC 2516: A Method for Transmitting PPP Over Ethernet (PPPoE). Internet Engineering Task Force (IETF), 1999. https://tools.ietf.org/ html/rfc2516

[38] J. Mauch. openntpproject: NTP Scanning Project. http://openntpproject. org/

[39] D. Menscher. NTP issues today. Outages Mailing List http: //mailman.nanog.org/pipermail/nanog/2012-November/053494.html November 2012.

[40] Microsoft. MS05-019: Vulnerabilities in TCP/IP could allow remote code execution and denial of service, 2010. https://support.microsoft. com/en-us/kb/893066 (Accessed July 2015).

[41] I. Miller. RFC 3128 (Informational): Protection Against a Variant of the Tiny Fragment Attack. Internet Engineering Task Force (IETF), 2001. https://tools.ietf.org/html/rfc3128

[42] D. Mills, J. Martin, J. Burbank, and W. Kasch. RFC 5905: Network Time Protocol Version 4: Protocol and Algorithms Specification. Internet Engineering Task Force (IETF), 2010. http://tools.ietf.org/html/rfc5905

[43] D. L. Mills. Computer Network Time Synchronization. CRC Press, 2nd edition, 2011.

[44] N. Minar. A survey of the NTP network, 1999.

[45] T. Mizrahi. RFC 7384 (Informational): Security Requirements of Time Protocols in Packet Switched Networks. Internet Engineering Task Force (IETF), 2012. http://tools.ietf.org/html/rfc7384

[46] M. Morowczynski. Did your active directory domain time just jump to the year 2000? Microsoft Server \& Tools Blogs http://blogs.technet.com/b/askpfeplat/archive/2012/11/19/ did-your-active-directory-domain-time-just-jump-to-the-year-2000. aspx November 2012.

[47] C. D. Murta, P. R. Torres Jr, and P. Mohapatra. Characterizing quality of time and topology in a time synchronization network. In GLOBECOM, 2006.

[48] P. Mutton. Certificate revocation: Why browsers remain affected by heartbleed. http://news.netcraft.com/archives/2014/04/24/ certificate-revocation-why-browsers-remain-affected-by-heartbleed. html April 242014.

[49] National Vulnerability Database. CVE-2014-9295: Multiple stack-based buffer overflows in ntpd in ntp before 4.2.8, 2014. https://web.nvd.nist. gov/view/vuln/detail?vulnId=CVE-2014-9295

[50] J. Novak. Target-based fragmentation reassembly. Technical report, Sourcefire, Incorporated, 2005.

[51] A. Peterson. Researchers say u.s. internet traffic was re-routed through belarus. that's a problem. Washington Post Blogs: The Switch, November 202013 .

[52] J. Postel. RFC 768: User Datagram Protocol. Internet Engineering Task Force (IETF), 1980. https://tools.ietf.org/html/rfc768

[53] J. Postel. RFC 791: INTERNET PROTOCOL: DARPA INTERNET PROGRAM PROTOCOL SPECIFICATION. Internet Engineering Task Force (IETF), 1981. https://tools.ietf.org/html/rfc791

[54] J. Postel. RFC 792: INTERNET CONTROL MESSAGE PROTOCOL. Internet Engineering Task Force (IETF), 1981. https://tools.ietf.org/ html/rfc792

[55] T. H. Ptacek and T. N. Newsham. Insertion, evasion, and denial of service: Eluding network intrusion detection. Technical report, Secure Networks, Inc., 1998.

[56] S. Röttger. Analysis of the ntp autokey procedures. Master's thesis, Technische Universitt Braunschweig, 2012.

[57] S. Rottger. Finding and exploiting ntpd vulnerabilities, $2015 . \quad$ http://googleprojectzero.blogspot.co.uk/2015/01/ finding-and-exploiting-ntpd.html

[58] S. Santesson, M. Myers, R. Ankney, A. Malpani, S. Galperin, and C. Adams. RFC 6960: X.509 Internet Public Key Infrastructure Online Certificate Status Protocol OCSP. Internet Engineering Task Force (IETF), 2013. https://tools.ietf.org/html//rfc6960

[59] C. Schramm. Why does Linux enforce a minimum MTU of 552?, 2012. http://cschramm.blogspot.com/2012/12/ why-does-linux-enforce-minimum-mtu-of.html

[60] J. Selvi. Bypassing HTTP strict transport security. Black Hat Europe, 2014.

[61] J. Selvi. Breaking SSL using time synchronisation attacks. DEFCON'23, 2015.

[62] U. Shankar and V. Paxson. Active mapping: Resisting NIDS evasion without altering traffic. In Symposium on Security and Privacy, pages 44-61. IEEE, 2003.

[63] D. Sibold, S. Roettger, and K. Teichel. draft-ietf-ntpnetwork-time-security-10: Network Time Security. Internet Engineering Task Force (IETF), 2015. https://tools.ietf.org/html/ draft-ietf-ntp-network-time-security-10

[64] H. Stenn. Antw: Re: Proposed REFID changes. NTP Working Group Mailing List http://lists.ntp.org/pipermail/ntpwg/2015-July/ 002291.html July 2015.

[65] H. Stenn. NTP's REFID. http://nwtime.org/ntps-refid// September 2015.

[66] H. Stenn. Securing the network time protocol. Communications of the ACM: ACM Queue, 13(1), 2015.

[67] P. Vixie. Rate-limiting state. Communications of the ACM: ACM Queue, 12(2):10, 2014.

[68] P. Vixie and V. Schryver. DNS Response Rate Limiting (DNS RRL). http://ss.vix.su/ vixie/isc-tn-2012-1.txt April 2012.

[69] VMware. Timekeeping in vmware virtual machines: vsphere 5.0, workstation 8.0, fusion 4.0, 2011. http://www.vmware.com/files/pdf/ Timekeeping-In-VirtualMachines.pdf (Accessed July 2015).

[70] L. Zhang, D. Choffnes, D. Levin, T. Dumitras, A. Mislove, A. Schulman, and C. Wilson. Analysis of SSL certificate reissues and revocations in the wake of heartbleed. In Internet Measurement Conference (IMC'14), pages 489-502, 2014. 